\title{
Mobile Social Helping Platform of LBS
}

\author{
Chen Yuefeng ${ }^{1}, \quad$ Li Bingquan ${ }^{2}$, Gao sheng ${ }^{3 *}$ and Peng Linxi ${ }^{4 *}$ \\ 1,2,3* information school, Guangdong Ocean University, Guangdong \\ $4 *$ Department of Computer and Education Software, Guangzhou Univ., \\ Guangzhou \\ 1yuefengch71@sina.com, ${ }^{2}$ tosmq009@qq.com, ${ }^{3} 13924401222 @ 139 . c o m$, \\ flyingday@139.com
}

\begin{abstract}
Mobile Internet grows with demands of location information of mobile users . In order to overcome the limitation of sociality, we develop Mobile social helping platform (MSH) of $L B S$, which realize the sharing of sociality. First, we analyze the requirement of MSHP platform, then design its architecture and database, and finally discuss the key technologies of MSHP. The platform makes use of open source technology, implements and optimizes LBS service. The simulation results show the system has good portability and maintainability, which is easy to be commercialized.
\end{abstract}

Keywords: Location Based Service(LBS); Mobile social; Global Position System(GPS)

\section{Introduction}

With the development of the popularity of mobile phones and mobile Internet, the needs of personalized social have brought new challenges to the traditional instant communication software. Social applications based on LBS (location based service Location Based Service) become research spot in applications of intelligent mobile phone. LBS refers to that the mobile terminals obtain the current location information using various positioning technologies, and then receive service through wireless network[1,2]. Mobile providers support LBS services with built-in GPS module in mobile devices for applications, such as e-commerce, traffic management etc. Therefore for mobile providers, it is an important means of competition to develop mobile social application platform at present, and mobile social application has become the most popular application on the mobile platform.

The current social platform faces many challenges, for example, information security, privacy and life safety, affect the social software. The papers [3-4] make research on LBS of context-aware and the papers [5-6] contribute on mobile spatial information services based on LBS. The paper [7] discusses the structure of LBS, and a framework of webbased LBS is proposed in the paper [8]. But at present the LBS is always separately used in the mobile Internet, social networking or e-commerce. Therefore presently a comprehensive application platform becomes an important research field of mobile applications, which will bring great benefit to the social activities and the development of community culture.

\section{Introduce to MSHP Platform}

With the rapid development of Internet technology, mobile positioning terminal has become the main way for people to communicate and exchange of information with each other. With the help of wireless communication and network technology, Mobile Social Helping Platform (MSHP) based on LBS makes combination with electronic map, user location and user interested information, providing functions of goods transactions and 
social helping, and providing fresh experience for the user to take part in social activities or shopping.

The MSHP is a platform based on the Android, with which people can make friends and take team interaction through positioning technology of mobile phone. Locating friends mean that we can query the people according our specified distance and then choose them to chat. Team interaction is the real group activities. Users can publish (shopping spree, group purchase or travel) tasks, then other users nearing the specified range will search to the interest tasks, and participate in these tasks. The users can benefit from the group purchase price, but also be able to make more friends. The MSHP platform provides us more humane way of communication to promote the exchanges between people.

\section{Requirements Analysis of MSHP Platform}

\subsection{Foreground Functional Structure of MSHP}

In foreground of MSHP, it includes: friend management, task management and system management. Task management includes my task, task list and map labeling management; friends management module consists of add friend, delete friend, chat and query friend list; and system management module includes the system setting, user login and task setting. The foreground function structure is depicted as Figure 1.

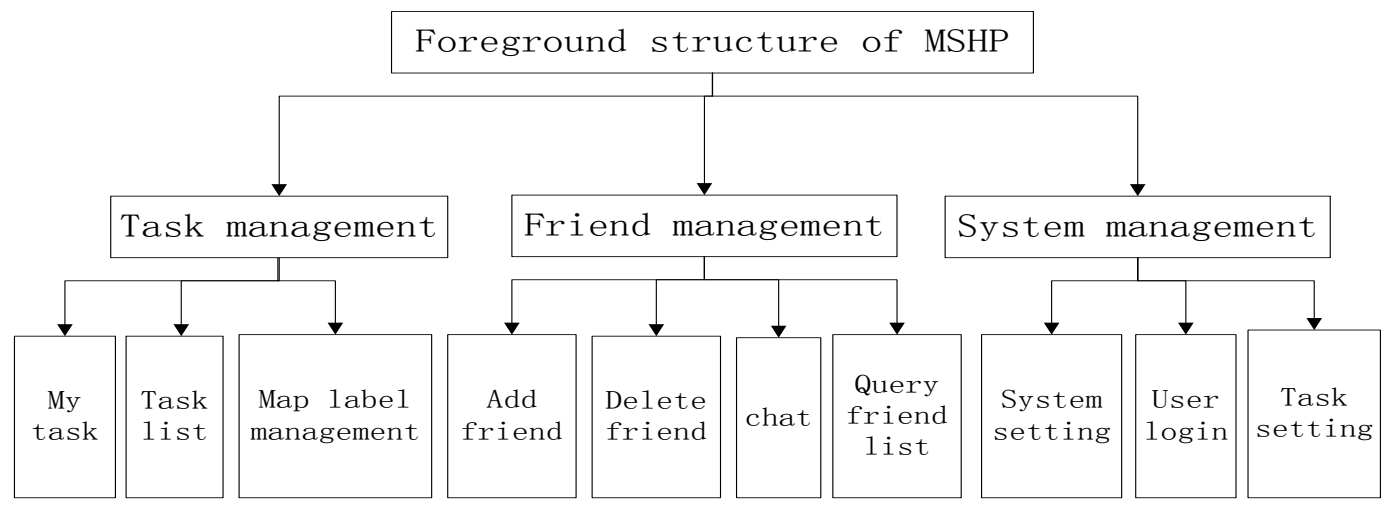

Figure 1. Foreground Function Structure of MSHP

\subsection{Background functional structure of MSHP}

Background function of MSHP includes report management, account management, authority management and task management.

1) The report consists of user report and task report management. From function of user report, we can query situations of user registration and login within a certain time, and then the system displays the query result in chart form or derives to the Excel file. Like the user report, from function of task report, we can query situations of published task.

2) In account management, we can access to the specified account information and then carry addition or deletion;

3) The authority management module provides functions of setting up and maintenance account permissions;

4) In task management, the administrator can make CRUD and audit operations to task. The illegal task will be denied.

The foreground function structure is depicted as Figure 2. 


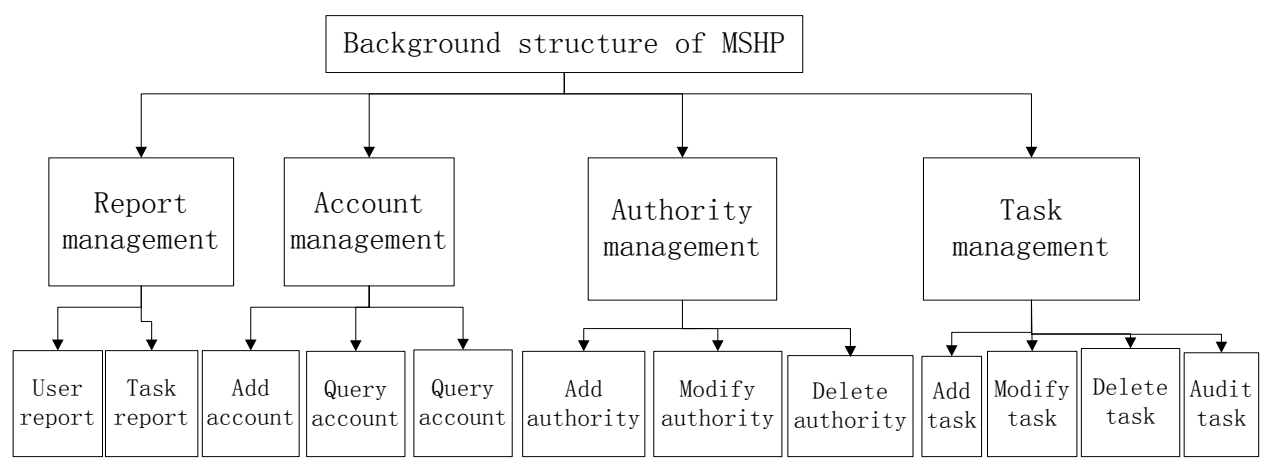

Figure 2. Background Function Structure of MSHP

\section{Design of MSHP}

\subsection{Architecture Design}

We design MSHP platform from the literature [9-11]. In the design of client, we implement the LBS services using Android application framework to call GPS locating module, JPush API and Baidu map API. We adopt Tomcat as server. When the client clicks the screen, the system sends request to the monitoring Service class, then Service class processes request and calls the HTTPClient class to assemble and send the request URL, and then receives asynchronous data to refresh interface. In server, the Strust2 framework receives URL requests to call the corresponding Action to process data, and then the Action calls Hibernate to persistent data. Lastly according to request call, it will call the pushing frame, assemble pushing message and realize entity class pushing. Architecture design is shown in Figure 3.

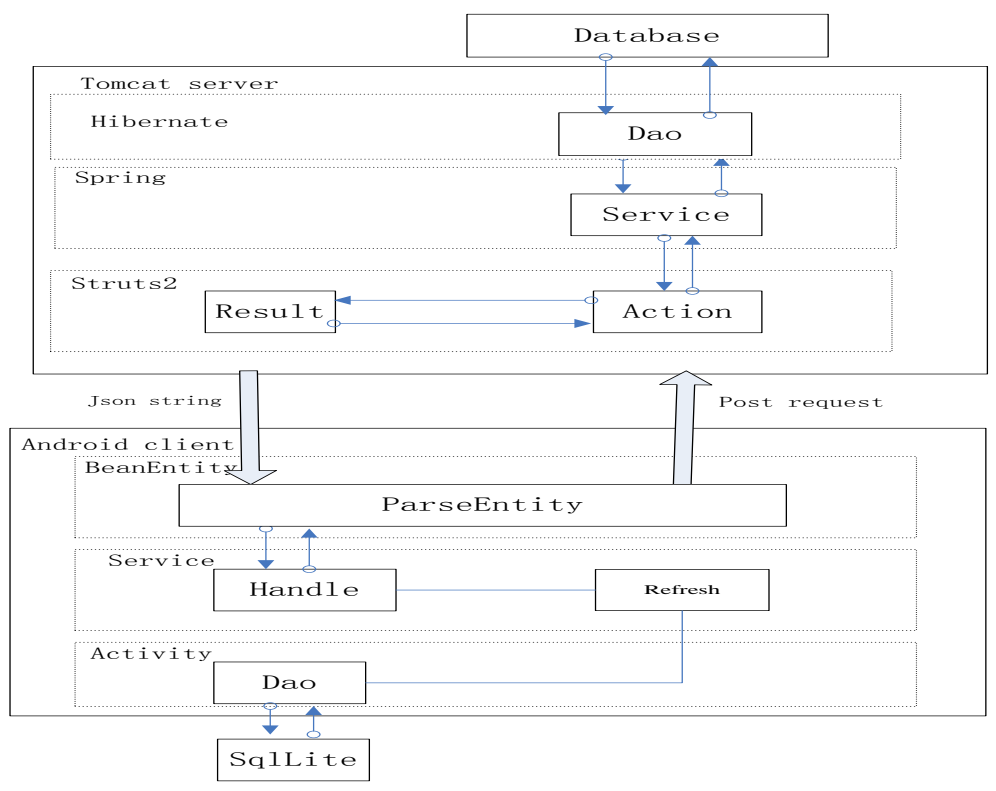

Figure 3. System Architecture

\subsection{Design of MSHP Platform Data Model}

From analyzing to the entities of MSHP platform, we design entities: user, activity, friend and task. And we had to design new entities to depict relationships of many to many association between some entities. These derived entities involve activity list, task list, chatting message and checking login list. Furthermore in order to improve the 
accessing speed, we separately store pictures related to activity, friend and task entity into pictures list. The ER (Entity relationship) model in server is shown in Figure 4.

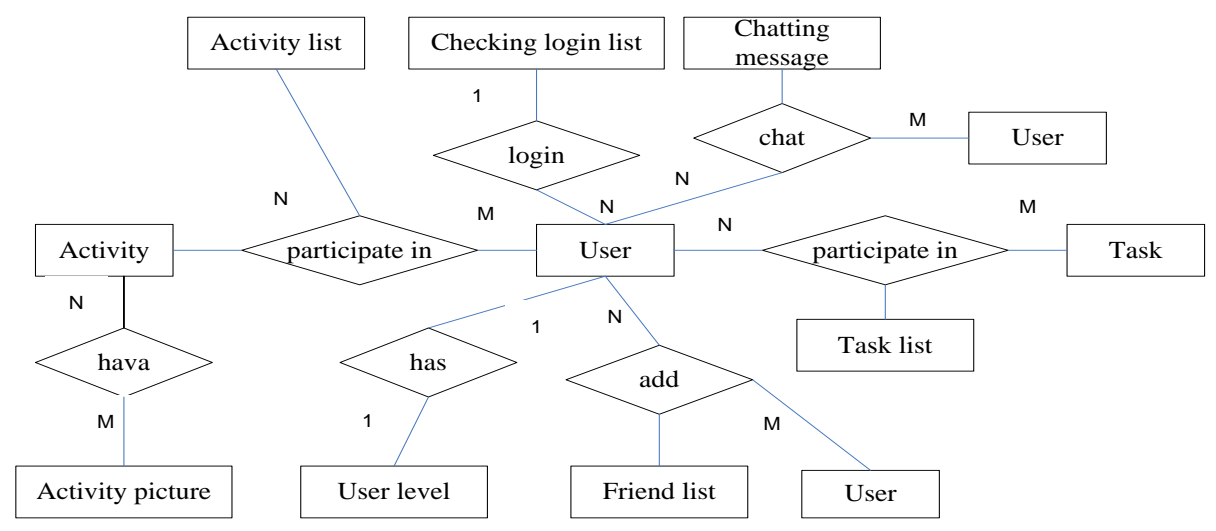

Figure 4. ER Diagram

\subsection{Design of Classes}

With a procedure of "user's access to business information and business pushs preferential information to the users" as an example, we mainly design the following entity classes: User class, Location class, Business class, Category class and PreferentialInfo class. The relationships of these classes are shown as Figure 5.

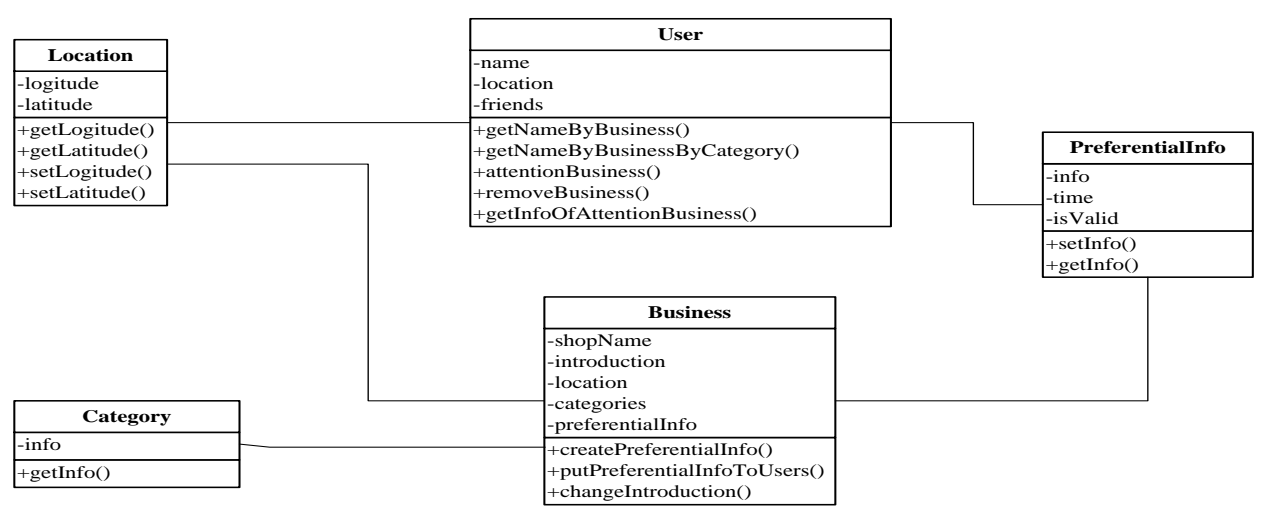

Figure 5. Class Relationship of User with Business

\subsection{Key Techniques of MSHP}

4.4.1. Operation Architecture of Server: In MSHP, we establish server using Nginx+Tomcat framework: Nginx is used to process the static file and make the reversion proxy; Tomcat is used to process accessing for business logic. In order to solve the congestion of Internet network and improve the response speed of user access to the site, we adopt the scheme of content delivery network (CDN). By placing the node servers throughout the network, the system can really redirect the user to the nearest service node according to the user request.

In our design, MSHP should support hundreds of thousands accessing in one day. And we regularly make database backup every day, to ensure that the time logs are consistency with timing backup databases. With good scalability in MSHP, we enhance the numbers of Tomcat server to meet business needs when accessing pressure increase sharply. When building cluster, we need to setup parameter session for load balancing server. 
4.4.2. Localization: The core development of LBS application is localization technology. In Android, there are three map localization technology -- base station, network and GPS. We obtain user position by use of these three kinds of localization methods in MSHP. First we obtain probable position information through localization of network, then obtain a precise geographic coordinates through the GPS. Lastly we acquire position by the base station method if GPS fails to get that due to timeout. Geographic location services mainly include GPS data and real-time address, which is an important part of geographic coding and real time location [12]. The mainly class is Geocoder and LocationManager class. The steps of GPS algorithm are described as follows:

1)Calls the getSystemService() method to get the LocationManager object;

2)Calls locationManager.getProvider() method to get the best Provider object;

3)Calls locationManager.getLastKnownLocation() method to get the last position information;

4)When the position information is null, call locationManager.requestLocationUpdates() method to update the location information;

5) Calls locationManager. addGpsStatusListener() method to increase listener of the GPS state;

6) If the last position information is empty, then displays the default longitude, otherwise displays the new position and repeat steps 1 6.

4.4.3. Pushing technology: For the client of the MSHP platform is device with limited resource, thus we must ensure the timeliness of data in the design, but also consider how to save power and traffic as far as possible. The common methods to obtain updated information on the server are Polling and Pushing. Polling means dropping, namely query the data of the server regularly; Pushing is namely that the server will push the real-time data to the client. In MSHP, we adopt pushing technology- Androidpn (Android push notification), which is open source implementation of Java based on XMPP (extensible messaging and Presence Protocol), including the client and server-side complete [12].

4.4.4. Map API: When the business issues preferential information, need to call map API to label its address. After contrasting several popular map API - Baidu, Google, MAPABC and mapbar, the MSHP chooses Baidu map API. Google and Baidu API map are the best choices on the usability. But Google map API has to use corrective algorithm to correct the signal interference. Therefore for mobile application developer in China, Baidu map API can not only provide more accurate location information, but also is free of charge and without the use of correction algorithm.

4.4.5. UI (user interface) design: Although the Android platform provides the UI function, but it is difficult to design a friendly, personalized interface. We design userdefined controls through combining several standard controls or modifing the properties of standard controls.

User-defined controls can be obtained by the following steps:

1) Establish a XML file to define attributes of the control;

2) Define a class of user-defined control inheriting from the View class, and connects variable of the class with properties in the XML file;

3) Define the control class to the XML file for layout;

4) Invocate the corresponding method of the user-defined control objects in the interface to obtain the desired interface.

4.4.6. Strategy of memory management: Memory allocation is realized by calling malloc and free method in tradition. It is simple, but may lead to memory fragmentation and aggravate the burden of operating system memory manager. Therefore, for data with 
high accessing rate, we use Memcached as distributed cache technology. In distributing and managing memory, Memcached divides the allocated memory into certain length of block thereby solving the memory fragmentation.

\section{Conclusion}

With the fundamental functions of LBS, the MSHP can be embedded into other LBS services and provide a convenient, flexible interactive way for communication, making friends, shopping and other social activities. Moreover, the platform provides a good user experience with the advantages of simple operation, beautiful interface. After perfecting, the platform can be widespread used in commerce. The next research direction of this application is to utilize the privacy protection technology and prevent the disclosure of sensitive information through data encryption or limited release means.

\section{Acknowledgements}

This research was supported by the National Natural Science Foundation of China (61100150), and Research Plan of Guangzhou Education Bureau (2012A077), and Students Entrepreneurship Training Project of Guangdong Ocean University (1056613096).

\section{References}

[1] B. Jing and X. B. Yao, "Location based service and GIS in perspective computers", Environment and Urban system, vol. 30, no. 6, (2006).

[2] A. Zipf, "User-Adaptive Maps for Location-Based Services (LBS) for Tourism", European Media Laboratory-EML, Heidelberg, Germany, (2002), pp. 1-2.

[3] S. Gessler, M. Martin and S. Weiss, "Context Awareness in Future Life Scenarios: Impact on Service Provisioning Platforms", Applications and the Internet Workshops, (2005); Saint, Canada.

[4] K. Rehman, F. Stajano and G. Coulouris, "An Architecture for Interactive Context-Aware Applications", IEEE Pervasive Computing, vol. 6, no. 1, (2007).

[5] T. D. Roza and G. Bilchev, "An overview of Location-Based Services”, BT technology Journal, vol. 21, no. $1,(2003)$.

[6] J. Schiler and H. Voisarda, "Location-Based Services", Morgan Kaufmann, San Francico, vol. 4, (2004), pp.112-124.

[7] J. Rui, M. Adriano and R. Helena, "The Around Architecture for Dynamic Location-Based Services", Mobile Networks and Applications, vol. 8, (2003), pp. 377-387.

[8] D. Jacob, T. Tele and V. Bert, "Design of a Web-Based LBS Framework Addressing Usability, Cost, and Implementation Constraints", World Wide Web, vol. 13, (2010), pp. 391-418.

[9] W. Chung and C. Samd, "An accurate ultra wideband ranging for preciseion asset location", Proceedings of Ultra wideband System and Technologies, (2003); Reston, USA.

[10] S. Havard and S. Torbjorn, "Location Based Services in mobile Java applications: A comparative study of Java Micro Edition and Android", Buskerud University College Kongsberg, (2008); Norway.

[11]R. Jose, A. Moreria and H. Rodrigus, "The Around Architecture for Dynamic Location-Based Services", Mobile Networks and Applications, vol. 8, (2003).

[12] R. Filjar, L. Busic, S. Desic and D. Huljenic, "LBS Position Estimation by Adaptive Selection of Positioning Sensors Based on Requested QoS”, Computer Science, (2008).

\section{Author}

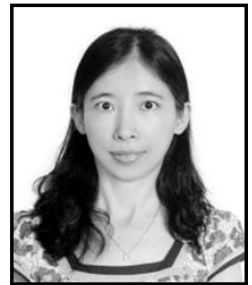

Yuefeng Chen is an associate professor of Computer Science at the Guangdong Ocean University, Guangdong. She received her Master in Communication and information engineering in South China University of Technology. Her current research focuses on mobile programming, artifical immune, algorithm design and analysis, network security, etc. 